\title{
DNA methylation of GFI1 as a mediator of the association between prenatal smoking exposure and ADHD symptoms at 6 years: the Hokkaido Study on Environment and Children's Health
}

Kunio Miyake ${ }^{1 *+} @$, Chihiro Miyashita ${ }^{2 \dagger}$, Atsuko Ikeda-Araki ${ }^{2}$, Ryu Miura ${ }^{2}$, Sachiko Itoh ${ }^{2}$, Keiko Yamazaki $^{2}$, Sumitaka Kobayashi ${ }^{2}$, Hideyuki Masuda $^{2}$, Tadao Ooka ${ }^{1}$, Zentaro Yamagata ${ }^{1}$ and Reiko Kishi ${ }^{2}$

\begin{abstract}
Background: Prenatal smoking exposure has been associated with childhood attention-deficit/hyperactivity disorder (ADHD). However, the mechanism underlying this relationship remains unclear. We assessed whether DNA methylation differences may mediate the association between prenatal smoking exposure and ADHD symptoms at the age of 6 years.

Results: We selected 1150 mother-infant pairs from the Hokkaido Study on the Environment and Children's Health. Mothers were categorized into three groups according to plasma cotinine levels at the third trimester: non-smokers $(\leq 0.21 \mathrm{ng} / \mathrm{mL})$, passive smokers $(0.21-11.48 \mathrm{ng} / \mathrm{mL})$, and active smokers $(\geq 11.49 \mathrm{ng} / \mathrm{mL})$. The children's ADHD symptoms were determined by the ADHD-Rating Scale at the age of 6 years. Maternal active smoking during pregnancy was significantly associated with an increased risk of ADHD symptoms (odds ratio, 1.89; 95\% confidence interval, 1.143.15) compared to non-smoking after adjusting for covariates. DNA methylation of the growth factor-independent 1 transcriptional repressor (GF/1) region, as determined by bisulfite next-generation sequencing of cord blood samples, mediated $48.4 \%$ of the total effect of the association between maternal active smoking during pregnancy and ADHD symptoms. DNA methylation patterns of other genes (aryl-hydrocarbon receptor repressor [AHRR], cytochrome P450 family 1 subfamily A member 1 [CYP1A1], estrogen receptor 1 [ESR1], and myosin IG [MYO1G]) regions did not exert a statistically significant mediation effect.
\end{abstract}

Conclusions: Our findings demonstrated that DNA methylation of GF/1 mediated the association between maternal active smoking during pregnancy and ADHD symptoms at the age of 6 years.

Keywords: ADHD, Birth cohort, DNA methylation, DOHaD, GF/1, Prenatal smoking exposure

*Correspondence: kmiyake@yamanashi.ac.jp

†Kunio Miyake and Chihiro Miyashita contributed equally to this work ${ }^{1}$ Departments of Health Sciences, Interdisciplinary Graduate School of Medicine and Engineering, University of Yamanashi, 1110 Shimokato, Chuo, Yamanashi 409-3898, Japan

Full list of author information is available at the end of the article

\section{Background}

The concept of Developmental Origins of Health and Disease $(\mathrm{DOHaD})$ suggests that exposure to environmental stressors during prenatal and early postnatal periods increases susceptibility to adverse health outcomes later in life. It is particularly well known that prenatal smoking 
exposure can cause adverse health effects not only at birth, but also in the long term after birth. For instance, prenatal smoking exposure increases the risk of several adverse birth outcomes, including infant death [1], preterm birth [2], and low birth weight [3, 4]. Furthermore, prenatal smoking exposure has been associated with child health implications, including obesity [5], asthma [6], and neurodevelopmental disorders such as antisocial behavior, conduct disorder, pervasive developmental disorder (PDD), and attention-deficit/hyperactivity disorder (ADHD) $[7,8]$.

DNA methylation, one of the epigenetic modifications, plays an important role in the regulation of gene expression. DNA methylation patterns are erased after fertilization and re-established during embryonic development [9]. DNA methylation is susceptible to several environmental factors, particularly during fetal life $[10,11]$. In recent studies, prenatal smoking exposure has been associated with DNA methylation changes in cord blood. Previous epigenome-wide association studies (EWAS) have identified CpG sites of several genes, including arylhydrocarbon receptor repressor $(A H R R)$, cytochrome P450 family 1 subfamily A member 1 (CYP1A1), growth factor-independent 1 transcriptional repressor (GFI1), and myosin IG (MYO1G), that are sensitive to maternal smoking exposure [12-14]. Several studies have reported that DNA methylation changes are involved in prenatal smoking exposure and low birth weight. DNA methylation differences of several genes, including AHRR, GFI1, and $E X O C 2$, are significant mediators between prenatal smoking and low birth weight [15-17]. In contrast, there remain few reports regarding the effects of DNA methylation on the association between prenatal smoking exposure and postnatal outcomes.

ADHD is a complex disease that interacts with genetic and environmental factors. In addition to genetic approaches, recent studies have identified the epigenetic alterations involved in ADHD. Several CpG sites that are differentially methylated between cases and controls have been identified by candidate-gene and EWAS approaches [18-20]. In addition, two birth cohort studies have reported an association between DNA methylation at birth and childhood ADHD. Walton et al. [21] demonstrated that DNA methylation at birth relates to later ADHD symptom trajectories, although such an association was not observed at the age of 7 years. Gervin et al. [22] demonstrated that long-term prenatal exposure to paracetamol (acetaminophen) is associated with DNA methylation differences in children diagnosed with ADHD. Prenatal factors such as maternal substance (e.g., tobacco, alcohol, and maternal anti-depressant use), exposure to environmental chemicals (e.g., mercury, phthalates, and polyfluoroalkyl chemicals), and maternal physical and mental health (e.g., stress, depression, obesity, and thyroid dysfunction) have been reported to increase the risk of ADHD in children. In particular, there is strong evidence supporting an association between maternal smoking exposure during pregnancy and ADHD in children [23]. However, no studies have revealed whether DNA methylation mediates the association between maternal smoking during pregnancy and childhood ADHD symptoms.

This study aimed to explore the association among prenatal smoking exposure, ADHD symptoms at preschool age, and cord blood DNA methylation using a prospective birth cohort study, the Hokkaido Study on Environment and Children's Health. We have previously identified the CpG sites in which cord blood DNA methylation is altered by maternal smoking during pregnancy using the Illumina Infinium HumanMethylation450 BeadChips [24]. In this study, we selected the CpG sites of five genes (AHRR, CYP1A1, estrogen receptor 1 [ESR1], $M Y O 1 G$, and GFI1) whose DNA methylation was significantly altered by maternal smoking during pregnancy; DNA methylation rates were measured using bisulfite next-generation sequencing. Next, we evaluated whether DNA methylation differences in these genes mediated the association between prenatal smoking exposure and ADHD symptoms.

\section{Results}

Maternal cotinine levels are represented in Table 1. Of the 1150 mothers, 612 (53.2\%) were categorized as nonsmokers, $429(37.3 \%)$ as passive smokers, and $109(9.5 \%)$ as active smokers. There were $188(16.3 \%)$ children with ADHD symptoms. Maternal age, parity, education, household income, and ADHD symptoms were significantly different among the three categories. The birth weight of infants of active smokers was lower than that of infants of non-smokers or passive smokers.

Table 2 summarizes the results of univariate and multivariate analyses on the association between maternal smoking exposure during pregnancy and child ADHD symptoms at the age of 6 . Maternal active smoking during pregnancy was significantly associated with an increased risk of childhood ADHD symptoms in univariate (odds ratio [OR], 2.04; 95\% confidence interval [CI], 1.25-3.34) and multivariate (OR, 1.89; 95\% CI 1.14-3.15) logistic regression analyses. No significant association was observed between maternal passive smoking during pregnancy and ADHD symptoms.

Based on our previous EWAS results, we focused on the DNA methylation of five genes (AHRR, CYP1A1, ESR1, GFI1, and MYO1G) associated with prenatal smoke exposure. The analyzed regions of five genes are shown in Additional file 1: Fig. S1. Since the methylation 
Table 1 Study population characteristics according to maternal cotinine levels

\begin{tabular}{|c|c|c|c|c|c|}
\hline & \multirow{3}{*}{$\begin{array}{l}\text { All } \\
\overline{(n=1150)}\end{array}$} & \multicolumn{3}{|c|}{ Maternal cotinine (ng/ml) } & \multirow[t]{3}{*}{$P$ value } \\
\hline & & Non-smokers & Passive smokers & Active smokers & \\
\hline & & $(n=612)$ & $(n=429)$ & $(n=109)$ & \\
\hline \multicolumn{6}{|l|}{ Mothers } \\
\hline Age (years) & $31.3 \pm 4.4$ & $31.9 \pm 4.2$ & $30.5 \pm 4.5$ & $31.2 \pm 4.6$ & $<0.001$ \\
\hline $\mathrm{BMI}\left(\mathrm{kg} / \mathrm{m}^{2}\right)$ & $21.1 \pm 3.1$ & $21.2 \pm 3.2$ & $20.9 \pm 2.8$ & $21.5 \pm 4.0$ & 0.167 \\
\hline Parity & & & & & $<0.001$ \\
\hline Primiparous & $495(43.0)$ & $236(38.6)$ & $223(52.0)$ & $36(33.0)$ & \\
\hline Multiparous & $652(56.7)$ & $373(60.9)$ & $206(48.0)$ & $73(67.0)$ & \\
\hline Missing data & $3(0.3)$ & $3(0.5)$ & $0(0.0)$ & $0(0.0)$ & \\
\hline Alcohol intake during pregnancy & & & & & 0.061 \\
\hline No & $1016(88.3)$ & $551(90.0)$ & $375(87.4)$ & $90(82.6)$ & \\
\hline Yes & $134(11.7)$ & $61(10.0)$ & $54(12.6)$ & $19(17.4)$ & \\
\hline Education (years) & & & & & $<0.001$ \\
\hline$\leq 12$ & $468(40.7)$ & $216(35.3)$ & $182(42.4)$ & $70(64.2)$ & \\
\hline$>12$ & $681(59.2)$ & $395(64.5)$ & $247(57.6)$ & $39(35.8)$ & \\
\hline Missing data & $1(0.1)$ & $1(0.2)$ & $0(0.0)$ & $0(0.0)$ & \\
\hline Annual household income (million JPY) & & & & & 0.002 \\
\hline $5<$ & $743(64.6)$ & $368(60.1)$ & $295(68.8)$ & $80(73.4)$ & \\
\hline$\geq 5$ & $407(35.4)$ & $244(39.9)$ & $134(31.2)$ & $29(26.6)$ & \\
\hline \multicolumn{6}{|l|}{ Infants } \\
\hline Sex & & & & & 0.095 \\
\hline Male & $579(50.3)$ & $290(47.4)$ & $232(54.1)$ & $57(52.3)$ & \\
\hline Female & $571(49.7)$ & $322(52.7)$ & $197(45.9)$ & $52(47.7)$ & \\
\hline Birth weight (g) & $3070.2 \pm 366.6$ & $3065.0 \pm 358.3$ & $3118.2 \pm 353.9$ & $2910.7 \pm 415.5$ & $<0.001$ \\
\hline Gestational age (weeks) & & & & & 0.338 \\
\hline Preterm (<37 weeks) & $28(2.4)$ & $17(2.8)$ & $7(1.6)$ & $4(3.7)$ & \\
\hline Full-term ( $\geq 37$ weeks) & $1122(97.6)$ & $595(97.2)$ & $422(98.4)$ & $105(96.3)$ & \\
\hline ADHD symptoms & & & & & 0.011 \\
\hline No & $962(83.7)$ & $527(86.1)$ & $353(82.3)$ & $82(75.2)$ & \\
\hline Yes & $188(16.3)$ & $85(13.9)$ & $76(17.7)$ & $27(24.8)$ & \\
\hline
\end{tabular}

Data are presented as mean \pm standard deviation (SD) or number (\%)

$P$ values derived from the Welch's test or Chi-square test

Table 2 Association of maternal cotinine levels with ADHD symptoms

\begin{tabular}{lll}
\hline & $\begin{array}{l}\text { Crude } \\
\text { OR }(95 \% \mathrm{Cl})\end{array}$ & $\begin{array}{l}\text { Adjusted } \\
\text { OR }(95 \% \mathrm{Cl})\end{array}$ \\
\hline Non-smokers & Reference & Reference \\
Passive smokers & $1.33(0.95,1.87)$ & $1.17(0.82,1.66)$ \\
Active smokers & $\mathbf{2 . 0 4}(\mathbf{1 . 2 5}, \mathbf{3 . 3 4})$ & $\mathbf{1 . 8 9}(\mathbf{1 . 1 4 , 3 . 1 5})$
\end{tabular}

Adjusted for maternal age, family income, maternal alcohol consumption during pregnancy, parity, child sex, pre-pregnancy BMI

Bold text indicates a statistically significant difference with a $p$ value $<0.05$

changes due to smoking exposure changed in the same direction in all CpGs (defined as a region) in each amplicon, the average methylation in the region was also used as a methylation index. We assessed the association between maternal smoking exposure during pregnancy and DNA methylation rate (Fig. 1 and Additional file 1: Table S1). Multivariate analysis revealed a significant negative association between maternal smoking exposure during pregnancy and DNA methylation rates on a region of $A H R R(\beta=-0.29 ; 95 \% \mathrm{CI}-0.35$ to -0.24$)$ and GFI1 ( $\beta=-0.19 ; 95 \% \mathrm{CI}-0.25$ to -0.13$)$. Meanwhile, a significant positive association was observed between maternal smoking exposure during pregnancy and DNA methylation rates on a region of MYO1G ( $\beta=0.12$; $95 \%$ CI 0.06-0.18) and CYP1A1 ( $\beta=0.09 ; 95 \%$ CI 0.04-0.15). However, the methylation rates of CYP1A1 were significantly different before CpG5 (effect size: approximately 3\%) and after CpG6 (effect size: approximately 0.3\%) 


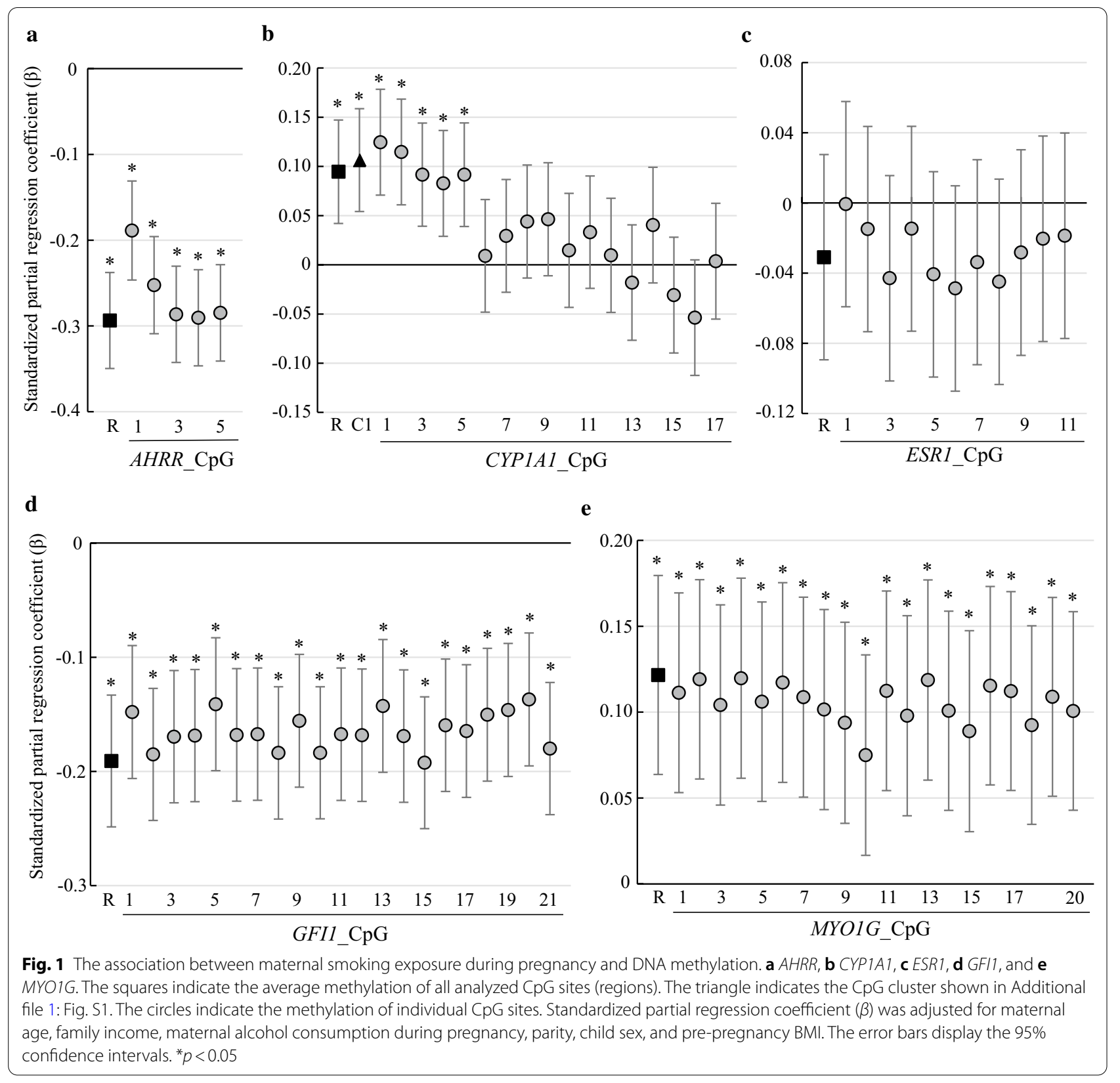

(Additional file 1: Fig. S2b). Therefore, CpG1 to CpG5 of CYP1A1 were analyzed as cluster 1 (Additional file 1: Fig. $\mathrm{S} 1 \mathrm{~b})$. The partial regression coefficient increased by 0.02 compared to the CYP1A1 region $(\beta=0.11 ; 95 \%$ CI $0.05-$ $0.16)$. No significant association was observed between maternal smoking exposure during pregnancy and DNA methylation rates both on a region and individual $\mathrm{CPG}$ site of ESR1.

Next, we examined the association between DNA methylation and childhood ADHD symptoms using logistic regression analysis. Figure 2 and Additional file 1:
Table S2 show the ORs and their 95\% CIs for the risk of ADHD symptoms in relation to DNA methylation. After adjustment for potential covariates, including maternal smoking exposure during pregnancy, a one-unit percent (\%) increase in DNA methylation rates on a region of ESR1 (OR, 0.93; 95\% CI 0.91-0.95) and GFI1 (OR, 0.94; 95\% CI 0.92-0.97) regions was associated with significantly lower odds of ADHD symptoms. No significant association was observed between DNA methylation rates on AHRR, CYP1A1, and MYO1G and childhood ADHD symptoms. Based on the results of the association 

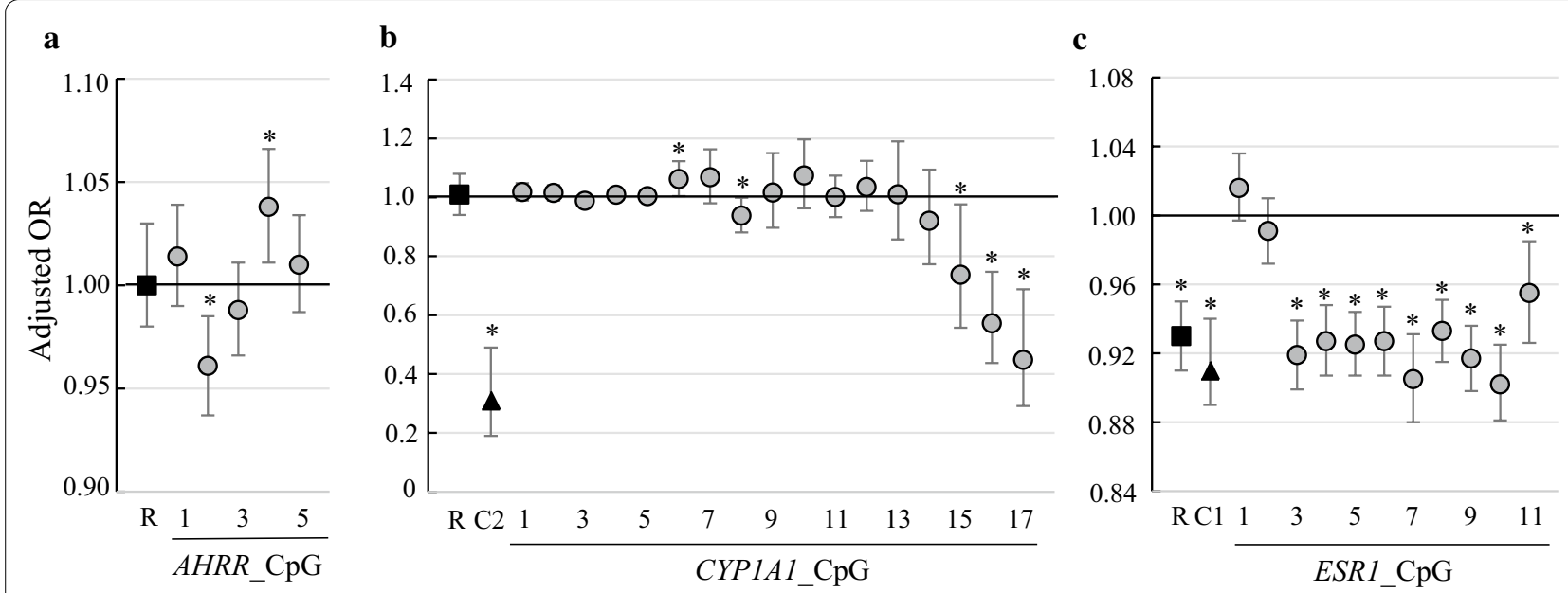

d
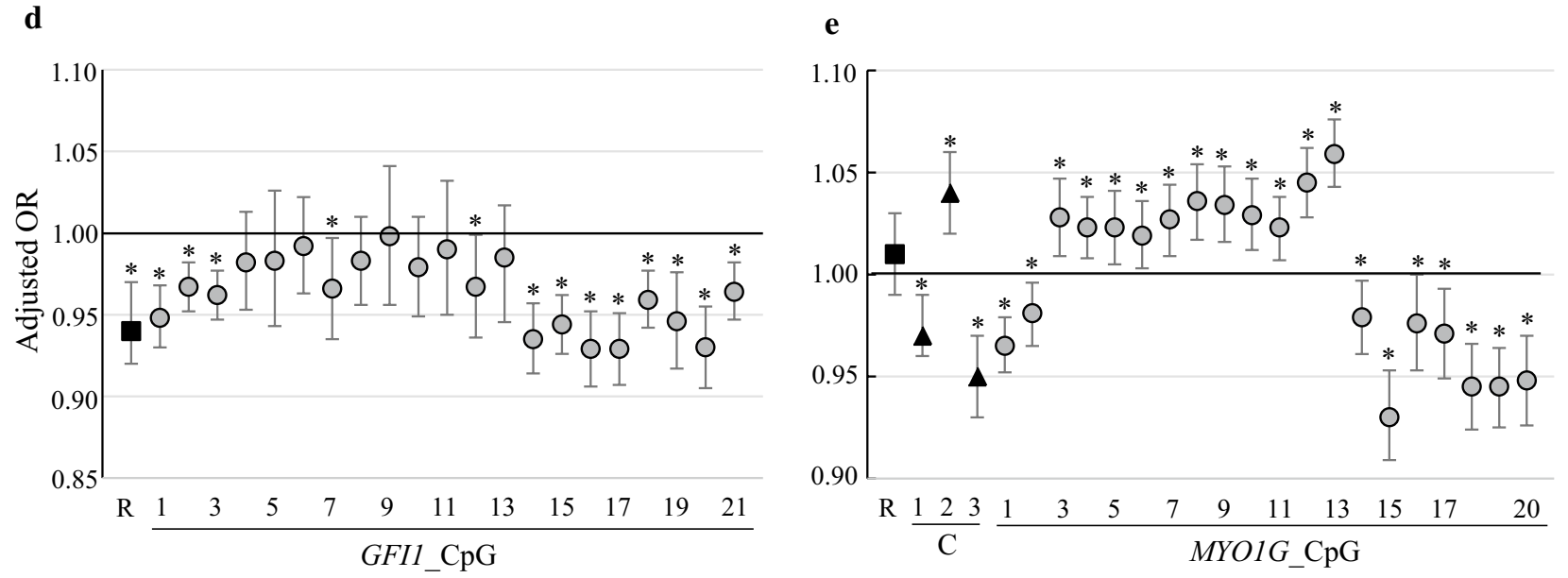

Fig. 2 Association of DNA methylation with ADHD symptoms by logistic regression analysis. a AHRR, b CYP1A1, c ESR1, d GFI1, and e MYO1G. Odds ratios were adjusted for maternal age, family income, maternal cotinine levels, maternal alcohol consumption during pregnancy, parity, child sex, and pre-pregnancy BMI. The squares indicate the average methylation of all analyzed $\mathrm{CpG}$ sites (regions). The triangle indicates the $\mathrm{CpG}$ cluster shown in Fig. S1. The circles indicate the methylation of individual CpG sites. The error bars display the $95 \%$ confidence intervals. ${ }^{*} p<0.05$

between individual CpG methylation and ADHD, we defined CpG clusters in CYP1A1 (Additional file 1: Fig. S1b), ESR1 (Additional file 1: Fig. S1c), and MYO1G (Additional file 1: Fig. S1e). When analyzed at a cluster level, a one-unit increase (\%) in the DNA methylation of CYP1A1 cluster 2 (OR, 0.31; 95\% CI 0.19-0.49), ESR1 cluster 1 (OR, 0.91; 95\% CI 0.89-0.94), and MYO1G clusters 1 and 3 (OR, 0.97; 95\% CI 0.96-0.99 and OR, 0.95; 95\% CI 0.93-0.97, respectively) was associated with significantly lower odds of ADHD symptoms. Meanwhile, a one-unit increase (\%) in the DNA methylation of $M Y O 1 G$ cluster 2 (OR, 1.04; 95\% CI 1.02-1.06) was associated with significantly higher odds of ADHD symptoms.

To elucidate whether DNA methylation changes mediated the association between prenatal smoke exposure and ADHD symptoms, a mediation analysis was performed. The results indicated that DNA methylation of the GFI1 region mediated $48.4 \%$ of the total effect of the association between maternal active smoking during pregnancy and ADHD symptoms (Fig. 3). DNA methylation of other genes regions did not exert a statistically significant mediation effect (Additional file 1: Table S3).

\section{Discussion}

The molecular mechanisms linking maternal smoking during pregnancy to childhood ADHD symptoms remain unclear. This is the first study showing that maternal active smoking during pregnancy may be associated with ADHD symptoms at preschool age through DNA methylation of GFI1.

In a previous study, using the Strengths and Difficulties Questionnaire (SDQ), we reported that maternal active smoking during pregnancy was significantly associated with an increased risk of total difficulties and 


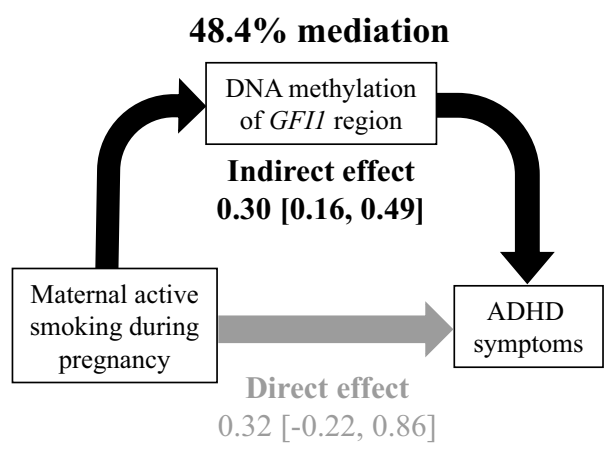

Fig. 3 Mediation of the association between active smoking during pregnancy and ADHD symptoms via DNA methylation of GFI1 regions. Mediation analysis was adjusted for maternal alcohol consumption during pregnancy, family income, pre-pregnancy $\mathrm{BMI}$, parity, gestational age, and infant sex. Percent mediation was calculated as the indirect effect derived by the total (indirect + direct) effect $\times 100 \%$

hyperactivity/inattention in 5-year-old children [25]. This study also revealed that maternal active smoking during pregnancy was significantly associated with an increased risk of ADHD in their children, based on the AttentionDeficit Hyperactivity Disorder-Rating Scale (ADHD-RS) at the age of 6 years. In contrast, the impact of passive smoking is controversial. In this study, no significant association was found between passive smoking during pregnancy and ADHD. Several epidemiological studies suggest that the association between pregnant mother smoking and ADHD is due to various confounding factors such as parental psychiatric history and social factors [26-28]. Our findings were consistent with those of previous studies showing that maternal smoking during pregnancy was associated with ADHD after adjusting for socioeconomic status. However, ADHD is characterized by a high inheritability ( $>70 \%)$ [29], but this study did not consider the parents' history of ADHD. Furthermore, it has been suggested that postnatal factors such as breastfeeding [30], second-hand smoke exposure [31], and maternal postnatal mental health [32] are associated with ADHD in children, but this study has not considered them.

In our previous study, we used a different cohort "Hokkaido Study Sapporo Cohort" to identify methylation site changes in cord blood due to maternal smoking exposure during pregnancy by a $450 \mathrm{~K}$ array and verified $A H R R_{-}$cg05575921 and CYP1A1_cg05549655 by bisulfite sequence using NGS [24]. The five CpGs of $A H R R$ in this study correspond to exactly the same sequence as that used in our previous report. The five CpGs of CYP1A1 analyzed in our previous study correspond to CpG1 to CpG5 in this study (Additional file 1:
Fig. S1b). With regard to these CpGs, similar methylation changes were confirmed in this study for all CpGs except CYP1A1_CpG4 (Additional file 1: Fig. S2b). In this study, the CpGs of GFI1 (cg12876356 and cg18146737) and MYO1G (cg12803068 and cg04180046) identified from the $450 \mathrm{~K}$ were confirmed to have similar methylation patterns in the NGS analysis. However, the CpGs of ESR1 (cg04063345 and cg15626350) and CYP1A1 (cg23727072 and cg00213123) did not match with the previous results. The possible reasons for this discrepancy are as follows: (1) Differences in methylation analysis methods (450 K array vs. bisulfite sequencing); (2) Differences in sample sizes (previous: 247, current: 1150); (3) Different groupings of maternal smoking exposure (previous: questionnaire, current: cotinine concentration); (4) Possible false positives in the $450 \mathrm{~K}$ analysis.

Wilmot et al. first reported the EWAS study which identified the genes with altered DNA methylation associated with ADHD using saliva samples, such as MYT1L and VIPR2 [18]. Furthermore, a large integrated genetic/epigenetic study using saliva samples reported an association between ADHD and DNA methylation at several novel CpG sites such as SLC7A8, MARK2, and SON [19]. A prospective study, the Avon Longitudinal Study of Parents and Children (ALSPAC), based on the EWAS approach identified 13 genes where the level of DNA methylation at birth and at age 7 years was significantly associated with differentiated ADHD trajectories between 7 and 15 years of age [21]. This study revealed that an increase in DNA methylation of ESR1 cluster 1 at birth was associated with significantly lower odds of ADHD symptoms at age 6 regardless of smoking exposure. ESR1, one of two ESR subtypes, is a nuclear receptor that is activated by the sex hormone estrogen. Single nucleotide polymorphisms within the ESR1 gene are associated with neuropsychiatric disorders including ADHD [33, 34]. According to animal studies, prenatal exposure to chemicals such as phthalates and benzophenone-3 impairs neurodevelopment via disruption of Esr expression and alteration of the epigenetic status [35, 36]. Our results suggest that DNA methylation of ESR1 may be a novel potential biomarker of ADHD symptoms. Increased DNA methylation of CYP1A1 cluster 2 at birth was also associated with significantly lower odds of ADHD symptoms at age 6. Since DNA methylation rates are as low as less than 1\% (Additional file 1: Fig. S2b), further verification is needed to determine if slight methylation changes in CYP1A1 are associated with ADHD symptoms.

This study showed that hypomethylation at GFI1 in umbilical cord blood could explain the positive association between maternal active smoking during pregnancy and childhood ADHD symptoms. In a study on children 
with ADHD, Sengapta et al. reported that maternal smoking exposure during pregnancy is associated with differences in GFI1 hypomethylation in childhood [37]. Hypomethylation at GFI1 mediates the effect of maternal smoking exposure during pregnancy on lower birth weight [15]. These findings suggest that maternal active smoking during pregnancy may cause hypomethylation at GFI1 and contribute to both low birth weight and ADHD symptoms during childhood. However, in this study, low birth weight did not significantly mediate the association between maternal smoking during pregnancy and childhood ADHD symptoms (data not shown). GFI1 is a transcriptional repressor that plays an important role in diverse developmental contexts such as hematopoiesis and oncogenesis. GFI1 is involved in the regulation of the $\mathrm{T}$ helper type 1 (Th1)-type immune response as well as the promotion of $\mathrm{T}$ helper type 2 (Th2) cell development [38]. ADHD has high comorbidity with both Th1and Th2-mediated disorders such as ear infections and atopic diseases [39]. There is indeed strong evidence that ADHD is associated with atopic diseases and that individuals suffering from atopic diseases have a $30-50 \%$ greater chance of developing ADHD [40]. However, it is not clear how GFI1 is involved in the molecular mechanisms of ADHD.

DNA methylation analysis by bisulfite sequence using a next-generation sequencer can clarify the methylation state of $\mathrm{CpG}$ around the probe of the methylation array. In addition to the methylation analysis of individual $\mathrm{CpGs}$, we analyzed the average methylation of all CpGs contained in the amplicon (defined as a region). It is also important to consider smoking- or ADHDassociated differentially methylated regions (DMR). The association among CYP1A1, ESR1, and MYO1G methylation and ADHD symptoms is clearly clustered within the region (Fig. 2b, c and e). In particular, DNA methylation in the $M Y O 1 G$ region showed no statistically significant mediating effect, but hypermethylation of $M Y O 1 G$ cluster 2 explained the positive association $(28.1 \%$ of the total effect) between active smoking during pregnancy and ADHD symptoms. In contrast, hypomethylation of MYO1G clusters 1 and 3 only partially explained the negative association between active smoking during pregnancy and ADHD symptoms. These results reveal that hypermethylation of $M Y O 1 G$ in active smokers during pregnancy is involved in both increased and weakened risk of ADHD symptoms. However, the amplicons in this study were arbitrarily designed to include methylation sites associated with maternal smoking exposure during pregnancy from a previous EWAS study. Therefore, the research is limited in that the interpretation of methylation in regions and clusters in this study may change depending on the methylation state around the amplicon.
It is well known that DNA methylation of promoter sequences acts to repress gene transcription. In contrast, gene-body DNA methylation has been reported to be associated with both gene activation and suppression [41, 42]. MYO1G regions analyzed in this study are located in a CpG island and exon 21 near the $3^{\prime}$ gene region. Hypermethylation in this gene region might correlate with active transcription of MYO1G [43, 44]. The region of GFI1 analyzed in this study is located in intron 3 and exon 4 (Additional file 1: Fig. S1d), and its effect on gene expression remains unknown. Therefore, genebody DNA methylation associated with ADHD should be examined together with gene expression levels.

Adverse environmental conditions during the fetal period to early childhood are linked to an increased risk of non-communicable diseases in adulthood. This concept is called DOHaD. Epigenetic modifications, such as DNA methylation, histone modification, and noncoding RNA, are thought to be molecular mechanisms of DOHaD. A limited number of studies have reported on outcomes other than birth weight. Parmar et al. [45] reported that DNA methylation at the GFI1 locus is associated with maternal prenatal smoking and cardiometabolic risk factors. Furthermore, long-term prenatal exposure to paracetamol (acetaminophen) is associated with DNA methylation differences in children diagnosed with ADHD [22]. To the best of our knowledge, this is the first study to clarify the association between prenatal smoking exposure and ADHD risk in children from the viewpoint of the molecular mechanism of $\mathrm{DOHaD}$.

This study has several limitations. First, ADHD suspected symptoms were not diagnosed but screened by ADHD-RS questionnaire; hence, there is a possibility of misclassification. However, previous studies have confirmed the reliability and validity of ADHD-RS for screening children in Japan [46, 47]. Second, umbilical cord blood-derived DNA methylation does not consider differences in cell composition. The EWAS applied several (reference-based and reference-free) methods for adjusting a difference in blood cell composition using a $450 \mathrm{~K}$ and EPIC array $[48,49]$. However, these methods are not directly applicable to candidate locus-specific DNA methylation analyses [37]. Smoking exposure can affect the cell type composition of blood. However, it has been reported that the difference in methylation due to smoking is greater than the difference in methylation between the two major pools of mononuclear cells (mainly lymphocytes) and granulocytes (mainly polymorphonuclear leukocytes) [50]. It is suggested that confounding by cell type is unlikely to rule out the results of smoking-related methylation differences.

Finally, DNA methylation patterns differ between tissues and cell types. We do not know whether the 
methylation changes in cord blood DNA also occur in brain tissue DNA. However, correlation of DNA methylation between blood and brain, and association between blood DNA methylation and brain phenotypes have been reported [51, 52]. Thus, DNA methylation in cord blood may serve as an indicator of neurodevelopmental disorders.

\section{Conclusions}

Our findings, taken together, have demonstrated that maternal active smoking during pregnancy was associated with altered DNA methylation and ADHD symptoms in children of preschool age. DNA methylation of GFI1 mediated the effect of maternal active smoking on ADHD symptoms.

\section{Methods}

\section{Study participants and baseline survey}

This prospective birth cohort study was a part of the Hokkaido Study on the Environment and Children's Health. The study design and procedures have been described previously in detail [53, 54]. Among all participants of 20,926 pregnant women who registered to the study from 2002 to 2012, this study comprised 3817 pregnant women and their offspring, delivered between March 2008 and April 2010. Pregnant women completed the baseline questionnaire, and peripheral blood sampling was conducted with prenatal health checkup by obstetricians; medical records and cord blood were taken at delivery. The questionnaire included maternal information about age, height, weight before pregnancy, parity, alcohol consumption during the first trimester, and annual household income. Birth weight, gestational age, and infant sex were obtained from medical records. A total of 1150 mother-infant pairs were finally included (Additional file 1: Fig. S3). The child's mother mainly filled out the questionnaire, including the ADHD survey for the child. In a few cases, when the mother withdrew from the study, the father or other guardian took over the responsibility to fill out the questionnaire. This study was conducted with informed consent of all subjects in writing. All procedures involving human subjects were approved by the University of Yamanashi, the Hokkaido University Graduate School of Medicine, and the Hokkaido University Center for Environmental and Health Science and were performed in accordance with relevant guidelines and regulations.

\section{Cotinine level measurement}

Plasma cotinine levels at the third trimester of pregnancy were measured using a highly sensitive enzyme-linked immunosorbent assay kit (Cosmic Corp., Tokyo, Japan). The detailed protocol has been described previously [55].
Based on our previous reports, which determined the cut-off levels for cotinine in non-smokers $(\leq 0.21 \mathrm{ng} / \mathrm{mL})$, passive smokers $(0.21-11.48 \mathrm{ng} / \mathrm{mL})$, and active smokers $(\geq 11.48 \mathrm{ng} / \mathrm{mL})$, we categorized the mothers into three groups.

\section{Follow-up and outcome assessment}

The ADHD-RS IV (home version) [56] was designed for assessment of two features of ADHD: inattention (nine items) and hyperactivity-impulsivity (nine items). We shared the Japanese version of the ADHD-RS IV via mail with 2735 participants and the parents completed the questionnaire to assess their child at the age of 6 . From the 1368 questionnaire responses collected until May 2016 (response rate 50.0\%), we calculated the total score by adding the scores of the inattention and hyperactivity-impulsivity scales. Tanaka concluded the Japanese version of the ADHD-RS IV shows good reliability and validity for screening children with possible ADHD [47]. We adopted Tanaka's cut-off criteria, namely, the $80^{\text {th }}$ percentile score for 5-7 yeas children (14 point for boys and 9.4 point for girls, respectively), to extract suspectADHD children.

\section{DNA methylation analysis}

Umbilical cord blood samples were acquired immediately after birth and stored at $-80{ }^{\circ} \mathrm{C}$. Genomic DNA was extracted from cord blood using a Maxwell ${ }^{\circledR}$ 16 DNA Purification Kit (Promega, Madison, WI, USA). DNA was subjected to bisulfite conversion by using an EZ DNA Methylation-Lightning Kit (Zymo Research, Irvine, CA, USA). Bisulfite-treated DNA was then amplified using FastStart Taq DNA Polymerase (Roche, Basel, Schweiz). Polymerase chain reaction (PCR) primers for bisulfite PCR were designed using MethPrimer (Additional file 1: Table S4). For nextgeneration sequencing, amplicon libraries were generated using an Ion Plus Fragment Library Kit (Thermo Fisher Scientific, MA, USA) as described previously [24]. Sequencing was performed using an Ion PGM Hi-Q View Sequencing Kit and 850 flows on an Ion 318 Chip Kit v2 (Thermo Fisher Scientific). After sequencing, single processing and base calling were performed using Torrent Suite 5.12.1 (Thermo Fisher Scientific). Methylation analysis was performed using Methylation Analysis_Amplicon plug-in v2.1 (Thermo Fisher Scientific). The analyzed regions of five genes are shown in Fig. 1. The number of CpG contained in the amplicon was 5 for $A H R R, 17$ for CYP1A1, 11 for ESR1, 21 for GFI, and 20 for MYO1G. The methylation rates of individual $\mathrm{CpGs}$ were used as a percentage. The methylation rates of each gene region were obtained by calculating the average of all CpGs in the amplicon. CpG 
clusters were defined as follows: in CYP1A1, CpG1 to CpG5 formed cluster 1 and CpG15 to CpG17 cluster 2 (Fig. 1b). In ESR, CpG3 to CpG11 were cluster 1 (Fig. 1c). In MYO1G, CpG1 and CpG2 were cluster 1, CpG3 to CpG13 cluster 2, and CpG14 to CpG20 cluster 3 (Fig. 1e). The methylation rates of each cluster were obtained by calculating the average of all CpGs in the cluster.

\section{Statistical analysis}

Based on a literature review of observational studies, maternal alcohol consumption during pregnancy, family income, pre-pregnancy body mass index (BMI), parity, gestational age, and infant sex were selected as possible confounders $[8,57]$. The association between maternal smoking during pregnancy and ADHD symptoms in children was examined using a logistic regression analysis adjusted for possible confounders. The association between maternal smoking during pregnancy and DNA methylation was examined by multiple linear regression analysis adjusted for possible confounders. The association between DNA methylation and ADHD symptoms in children was examined using logistic regression analysis adjusted for possible confounders and maternal smoking exposure during pregnancy.

Finally, a mediation analysis was used to estimate the degree of the association between prenatal smoking exposure and ADHD symptoms, which is explained by DNA methylation changes. The direct effect was the effect of the exposure $(\mathrm{X})$ on the outcome $(\mathrm{Y})$ at a fixed level of the mediator $(\mathrm{M})$. The indirect effect of $\mathrm{X}$ on $\mathrm{Y}$ through $\mathrm{M}$ can be quantified as the product of two coefficients: $a$ (the effect of $X$ on $M$ ) and $b$ (the effect of $M$ on $\mathrm{Y})$ pathways (i.e., ab). Percent mediation was calculated as the indirect effect divided by the total (indirect + direct) effect $\times 100 \%$. Mediation analysis included maternal alcohol consumption during pregnancy, family income, prepregnancy BMI, parity, gestational age, and infant sex as covariates. The bias-corrected and accelerated CIs of the indirect effect $(\mathrm{ab})$ were calculated by bootstrapping with 5000 iterations [58]. Mediation analysis was performed by using PROCESS version 3.5, a macro implemented in SPSS (IBM, Armonk, NY, USA). All statistical analyses were performed using SPSS version 27. A p-value of 0.05 (two-sided) was considered statistically significant.

\footnotetext{
Abbreviations

ADHD: Attention-deficit/hyperactivity disorder; ADHD-RS: Attention-Deficit Hyperactivity Disorder-Rating Scale; AHRR: Aryl-hydrocarbon receptor repressor; BMl: Body mass index; Cl: Confidence interval; CYP1A1: Cytochrome P450 family 1 subfamily A member 1; DOHaD: Developmental Origins of Health and Disease; ESR1: Estrogen receptor 1; EWAS: Epigenome-wide association study; GFI1: Growth factor-independent 1 transcriptional repressor; MYO1G:
}

Myosin IG; OR: Odds ratio; PCR: Polymerase chain reaction; SDQ: Strengths and Difficulties Questionnaire; Th1:T helper type 1; Th2: T helper type 2.

\section{Supplementary Information}

The online version contains supplementary material available at https://doi. org/10.1186/s13148-021-01063-z.

Additional file 1: Fig. S1. Base sequences analyzed by targeted bisulfite next-generation sequencing. Fig. S2. Comparison of methylated CpG sites among non-smokers, passive smokers, and active smokers. Fig. S3. Selection of the study population. Table S1. Association between maternal smoking during pregnancy and umbilical cord blood DNA methylation. Table S2. Association of umbilical cord blood DNA methylation with ADHD symptoms at 6 years of age. Table S3. Mediation analysis for the effect of DNA methylation in the association between active smoking during pregnancy and ADHD symptoms at 6 years of age. Table S4. List of bisulfite PCR primers.

\section{Acknowledgements}

We thank all the participants and staff of the Hokkaido Study on Environment and Children's Health. We would like to thank Editage (www.editage.com) for English language editing.

\section{Authors' contributions}

$\mathrm{KM}, \mathrm{CM}, \mathrm{RM}$, and RK conceived and designed the study. CM, AA, RM, SI, KY, SK, $\mathrm{HM}$, and RK collected the data. KM, CM, TO, and ZY performed the statistical analysis. KM, CM, RM, and HM performed the DNA methylation analysis. KM wrote the first draft of the manuscript. CM, RM, and ZY revised the manuscript. RK supervised the study. All authors read and approved the final manuscript.

\section{Funding}

This work was supported by a Grant-in-Aid for Health Science Research from the Japanese Ministry of Health, Labor and Welfare (No. JPMH17932352), a Grant-in-Aid for Scientific Research from the Japanese Ministry of Education, Culture, Sports, Science and Technology (No. JP16H02645; No. JP19H01071; No. JP20H03928; No. 20K21707), and the Environment Research and Technology Development Fund (No. JPMEERF20145054) from the Ministry of the Environment, Japan.

\section{Availability of data and materials}

The datasets used and analyzed during the present study are available from the corresponding author on reasonable request.

\section{Declarations}

\section{Ethics approval and consent to participate}

This study was conducted with informed consent of all subjects in writing. All procedures involving human subjects were approved by the University of Yamanashi, the Hokkaido University Graduate School of Medicine, and the Hokkaido University Center for Environmental and Health Science and were performed in accordance with relevant guidelines and regulations.

\section{Consent for publication}

Not applicable.

\section{Competing interests}

The authors declare that they have no competing interests.

\section{Author details}

${ }^{1}$ Departments of Health Sciences, Interdisciplinary Graduate School of Medicine and Engineering, University of Yamanashi, 1110 Shimokato, Chuo, Yamanashi 409-3898, Japan. ${ }^{2}$ Center for Environmental and Health Sciences, Hokkaido University, Hokkaido, Japan.

Received: 17 November 2020 Accepted: 25 March 2021

Published online: 07 April 2021 


\section{References}

1. Wisborg K, Kesmodel U, Henriksen TB, Olsen SF, Secher NJ. A prospective study of smoking during pregnancy and SIDS. Arch Dis Child. 2000;83:203-6.

2. Windham GC, Hopkins B, Fenster L, Swan SH. Prenatal active or passive tobacco smoke exposure and the risk of preterm delivery or low birth weight. Epidemiology. 2000;11:427-33.

3. Witt WP, Mandell KC, Wisk LE, Cheng ER, Chatterjee D, Wakeel F, et al. Infant birthweight in the US: the role of preconception stressful life events and substance use. Arch Womens Ment Health. 2016;19:529-42.

4. Kondracki AJ, Hofferth SL. A gestational vulnerability window for smoking exposure and the increased risk of preterm birth: how timing and intensity of maternal smoking matter. Reprod Health. 2019;16:43.

5. Qureshi R, Jadotte Y, Zha P, Porter SA, Holly C, Salmond S, et al. The association between prenatal exposure to environmental tobacco smoke and childhood obesity: a systematic review. JBI Database Syst Rev Implement Rep. 2018;16:1643-62.

6. Gilliland FD, Li YF, Peters JM. Effects of maternal smoking during pregnancy and environmental tobacco smoke on asthma and wheezing in children. Am J Respir Crit Care Med. 2001;163:429-36.

7. Tran PL, Lahti V, Lampi KM, Helenius H, Suominen A, Gissler M, et al. Smoking during pregnancy and risk of autism spectrum disorder in a Finnish National Birth Cohort. Paediatr Perinat Epidemiol. 2013;27:266-74.

8. Zhu JL, Olsen J, Liew Z, Li J, Niclasen J, Obel C. Parental smoking during pregnancy and ADHD in children: the Danish national birth cohort. Pediatrics. 2014;134:e382-8.

9. Messerschmidt DM, Knowles BB, Solter D. DNA methylation dynamics during epigenetic reprogramming in the germline and preimplantation embryos. Genes Dev. 2014;28:812-28.

10. Jirtle RL, Skinner MK. Environmental epigenomics and disease susceptibility. Nat Rev Genet. 2007:8:253-62.

11. Tran NQV, Miyake K. Neurodevelopmental disorders and environmental toxicants: epigenetics as an underlying mechanism. Int J Genomics. 2017;2017:7526592.

12. Joubert BR, Håberg SE, Nilsen RM, Wang X, Vollset SE, Murphy SK, et al. 450K epigenome-wide scan identifies differential DNA methylation in newborns related to maternal smoking during pregnancy. Environ Health Perspect. 2012;120:1425-31.

13. Richmond RC, Simpkin AJ, Woodward G, Gaunt TR, Lyttleton O, McArdle $W \mathrm{~L}$, et al. Prenatal exposure to maternal smoking and offspring DNA methylation across the lifecourse: findings from the Avon Longitudinal Study of Parents and Children (ALSPAC). Hum Mol Genet. 2015;24:2201-17

14. Lee KW, Richmond R, Hu P, French L, Shin J, Bourdon C, et al. Prenatal exposure to maternal cigarette smoking and DNA methylation: epigenome-wide association in a discovery sample of adolescents and replication in an independent cohort at birth through 17 years of age. Environ Health Perspect. 2015;123:193-9.

15. Küpers LK, Xu X, Jankipersadsing SA, Vaez A, la Bastide-van GS, Scholtens $S$, et al. DNA methylation mediates the effect of maternal smoking during pregnancy on birthweight of the offspring. Int J Epidemiol. 2015;44:1224-37.

16. Morales E, Vilahur N, Salas LA, Motta V, Fernandez MF, Murcia M, et al. Genome-wide DNA methylation study in human placenta identifies novel loci associated with maternal smoking during pregnancy. Int J Epidemiol. 2016;45:1644-55

17. Hannon E, Schendel D, Ladd-Acosta C, Grove J, Hansen CS, Hougaard DM, et al. Variable DNA methylation in neonates mediates the association between prenatal smoking and birth weight. Philos Trans R Soc Lond B Biol Sci. 2019:374:20180120.

18. Wilmot B, Fry R, Smeester L, Musser ED, Mill J, Nigg JT. Methylomic analysis of salivary DNA in childhood ADHD identifies altered DNA methylation in VIPR2. J Child Psychol Psychiatry. 2016;57:152-60.

19. Mooney MA, Ryabinin P, Wilmot B, Bhatt P, Mill J, Nigg JT. Large epigenome-wide association study of childhood ADHD identifies peripheral DNA methylation associated with disease and polygenic risk burden. Transl Psychiatry. 2020;10:8.

20. Meijer M, Klein M, Hannon E, van der Meer D, Hartman C, Oosterlaan J, et al. Genome-wide DNA methylation patterns in persistent attentiondeficit/hyperactivity disorder and in association with impulsive and callous traits. Front Genet. 2020;11:16.
21. Walton E, Pingault JB, Cecil CAM, Gaunt TR, Relton CL, Mill J, et al. Epigenetic profiling of ADHD symptoms trajectories: a prospective, methylome-wide study. Mol Psychiatry. 2017;22:250-6.

22. Gervin K, Nordeng H, Ystrom E, Reichborn-Kjennerud T, Lyle R. Long-term prenatal exposure to paracetamol is associated with DNA methylation differences in children diagnosed with ADHD. Clin Epigenetics. 2017;9:77.

23. Sciberras E, Mulraney M, Silva D, Coghill D. Prenatal risk factors and the etiology of ADHD-review of existing evidence. Curr Psychiatry Rep. 2017:19:1.

24. Miyake K, Kawaguchi A, Miura R, Kobayashi S, Tran NQV, Kobayashi S, et al. Association between DNA methylation in cord blood and maternal smoking: the Hokkaido Study on Environment and Children's Health. Sci Rep. 2018;8:5654

25. Minatoya M, Araki A, Itoh S, Yamazaki K, Kobayashi S, Miyashita C, et al. Prenatal tobacco exposure and ADHD symptoms at pre-school age: the Hokkaido Study on Environment and Children's Health. Environ Health Prev Med. 2019;24:74

26. López Seco F, Aguado-Gracia J, Mundo-Cid P, Acosta-García S, MartíSerrano S, Gaviria AM, et al. Maternal psychiatric history is associated with the symptom severity of ADHD in offspring. Psychiatry Res. 2015;226(2-3):507-12.

27. Dong T, Hu W, Zhou X, Lin H, Lan L, Hang B, et al. Prenatal exposure to maternal smoking during pregnancy and attention-deficit/hyperactivity disorder in offspring: a meta-analysis. Reprod Toxicol. 2018;76:63-70.

28. Froehlich TE, Anixt JS, Loe IM, Chirdkiatgumchai V, Kuan L, Gilman C. Update on environmental risk factors for attention-deficit/hyperactivity disorder. Curr Psychiatry Rep. 2011;13:333-44.

29. Faraone SV, Larsson H. Genetics of attention deficit hyperactivity disorder. Mol Psychiatry. 2019;24(4):562-75

30. Zeng Y, Tang Y, Tang J, Shi J, Zhang L, Zhu T, et al. Association between the different duration of breastfeeding and attention deficit/hyperactivity disorder in children: a systematic review and meta-analysis. Nutr Neurosci. 2020;23(10):811-23.

31. Huang A, Wu K, Cai Z, Lin Y, Zhang X, Huang Y. Association between postnatal second-hand smoke exposure and ADHD in children: a systematic review and meta-analysis. Environ Sci Pollut Res Int. 2021;28(2):1370-80.

32. Mulraney M, Giallo R, Efron D, Brown S, Nicholson JM, Sciberras E. Maternal postnatal mental health and offspring symptoms of ADHD at 8-9 years: pathways via parenting behavior. Eur Child Adolesc Psychiatry. 2019;28(7):923-32.

33. Pinsonneault JK, Frater JT, Kompa B, Mascarenhas R, Wang D, Sadee W. Intronic SNP in ESR1 encoding human estrogen receptor alpha is associated with brain ESR1 mRNA isoform expression and behavioral traits. PLOS ONE. 2017;12:e0179020.

34. Sun X, Wu Z, Cao Q, Qian Y, Liu Y, Yang B, et al. Genetic variant for behavioral regulation factor of executive function and its possible brain mechanism in attention deficit hyperactivity disorder. Sci Rep. 2018;8:7620.

35. Wnuk A, Rzemieniec J, Litwa E, Lasoń W, Kajta M. Prenatal exposure to benzophenone-3 (BP-3) induces apoptosis, disrupts estrogen receptor expression and alters the epigenetic status of mouse neurons. J Steroid Biochem Mol Biol. 2018:182:106-18.

36. Xu S, Zhang H, Pao PC, Lee A, Wang J, Chan YS, et al. Exposure to phthalates impaired neurodevelopment through estrogenic effects and induced DNA damage in neurons. Aquat Toxicol. 2020;222:105469.

37. Sengupta SM, Smith AK, Grizenko N, Joober R. Locus-specific DNA methylation changes and phenotypic variability in children with attentiondeficit hyperactivity disorder. Psychiatry Res. 2017;256:298-304.

38. Suzuki J, Maruyama S, Tamauchi H, Kuwahara M, Horiuchi M, Mizuki $M$, et al. Gfi1, a transcriptional repressor, inhibits the induction of the Thelper type 1 programme in activated CD4 T cells. Immunology. 2016;147:476-87.

39. Verlaet AAJ, Noriega DB, Hermans N, Savelkoul HFJ. Nutrition, immunological mechanisms and dietary immunomodulation in ADHD. Eur Child Adolesc Psychiatry. 2014:23:519-29.

40. Tsai JD, Chang SN, Mou CH, Sung FC, Lue KH. Association between atopic diseases and attention-deficit/hyperactivity disorder in childhood: a population-based case-control study. Ann Epidemiol. 2013;23:185-8.

41. Ball MP, Li JB, Gao Y, Lee JH, LeProust EM, Park IH, et al. Targeted and genome-scale strategies reveal gene-body methylation signatures in human cells. Nat Biotechnol. 2009;27:361-8. 
42. Aran D, Toperoff G, Rosenberg M, Hellman A. Replication timing-related and gene body-specific methylation of active human genes. Hum Mol Genet. 2011;20:670-80.

43. Zhu X, Li J, Deng S, Yu K, Liu X, Deng Q, et al. Genome-wide analysis of DNA methylation and cigarette smoking in a Chinese population. Environ Health Perspect. 2016;124:966-73.

44. Rzehak P, Saffery R, Reischl E, Covic M, Wahl S, Grote V, et al. Maternal smoking during pregnancy and DNA-methylation in children at age 5.5 years: epigenome-wide-analysis in the European Childhood Obesity Project (CHOP)-Study. PLoS ONE. 2016;11:e0155554.

45. Parmar P, Lowry E, Cugliari G, Suderman M, Wilson R, Karhunen V, et al. Association of maternal prenatal smoking GFI1-locus and cardio-metabolic phenotypes in 18,212 adults. EBioMedicine. 2018;38:206-16.

46. Takayanagi N, Yoshida S, Yasuda S, Adachi M, Kaneda-Osato A, Tanaka M et al. Psychometric properties of the Japanese ADHD-RS in preschool children. Res Dev Disabil. 2016;55:268-78.

47. Tanaka Y. Is ADHD symptom change according to the age from infancy to adolescence? Seishin shinkeigaku zasshi 2012;SS447-449.

48. Titus AJ, Gallimore RM, Salas LA, Christensen BC. Cell-type deconvolution from DNA methylation: a review of recent applications. Hum Mol Genet. 2017;26(R2):R216-24.

49. Houseman EA, Molitor J, Marsit CJ. Reference-free cell mixture adjustments in analysis of DNA methylation data. Bioinformatics. 2014;30(10):1431-9.

50. Joubert BR, Håberg SE, Bell DA, Nilsen RM, Vollset SE, Midttun O, et al. Maternal smoking and DNA methylation in newborns: in utero effect or epigenetic inheritance? Cancer Epidemiol Biomark Prev. 2014;23(6):1007-17.

51. Hannon E, Lunnon K, Schalkwyk L, Mill J. Interindividual methylomic variation across blood, cortex, and cerebellum: implications for epigenetic studies of neurological and neuropsychiatric phenotypes. Epigenetics. 2015;10:1024-32.

52. Jia T, Chu C, Liu Y, van Dongen J, Papastergios E, Armstrong NJ, et al. Epigenome-wide meta-analysis of blood DNA methylation and its association with subcortical volumes: findings from the ENIGMA Epigenetics Working Group. Mol Psychiatry. 2019. https://doi.org/10.1038/s41380-019-0605-z.

53. Kishi R, Kobayashi S, Ikeno T, Araki A, Miyashita C, Itoh S, et al. Ten years of progress in the Hokkaido birth cohort study on environment and children's health: cohort profile-updated 2013. Environ Health Prev Med. 2013;18:429-50.

54. Kishi R, Araki A, Minatoya M, Hanaoka T, Miyashita C, Itoh S, et al. The Hokkaido Birth Cohort Study on Environment and Children's Health: cohort profile-updated 2017. Environ Health Prev Med. 2017;22:46.

55. Sasaki S, Braimoh TS, Yila TA, Yoshioka E, Kishi R. Self-reported tobacco smoke exposure and plasma cotinine levels during pregnancy — a validation study in Northern Japan. Sci Total Environ. 2011;412-3:114-8.

56. DuPaul GJ, Power TJ, Anastopoulos AD, Reid R. ADHD rating scale-IV: checklists, norms, and clinical interpretation. New York: Guilford; 1998.

57. Burris HH, Baccarelli AA, Byun HM, Cantoral A, Just AC, Pantic l, et al. Offspring DNA methylation of the aryl-hydrocarbon receptor repressor gene is associated with maternal BMI, gestational age, and birth weight. Epigenetics. 2015;10:913-21.

58. Preacher KJ, Hayes AF. Asymptotic and resampling strategies for assessing and comparing indirect effects in multiple mediator models. Behav Res Methods. 2008:40(3):879-91.

\section{Publisher's Note}

Springer Nature remains neutral with regard to jurisdictional claims in published maps and institutional affiliations.
Ready to submit your research? Choose BMC and benefit from:

- fast, convenient online submission

- thorough peer review by experienced researchers in your field

- rapid publication on acceptance

- support for research data, including large and complex data types

- gold Open Access which fosters wider collaboration and increased citations

- maximum visibility for your research: over $100 \mathrm{M}$ website views per year

At BMC, research is always in progress.

Learn more biomedcentral.com/submissions 